\title{
EFFECTS OF ADAPTIVE INFORMATION PRESENTATION
}

\author{
Nicola Fricke \\ Technische Universität Berlin \\ Berlin, Germany \\ E-mail: Nicola.Fricke@zmms.tu-berlin.de
}

\begin{abstract}
Summary: Several new approaches to assisting the driver in dealing with the complex task of the driving situation are being developed. One possibility is to give support in the secondary task with the aim of facilitating the primary driving task. In this study, I tested whether a situation-dependent change of modality presentation for a secondary task can support time-sharing and enhance performance in the primary task. In an experiment, 42 subjects were engaged in a computer-simulated tracking task, which is a continuous task like driving a car. From time to time they also had to perform a decision-making task, which was to identify special numerical series. The task was an analogy to receiving a phone call while driving. The independent variables consisted of 1) demands of the situation (low vs. high) and 2) presentation modality of the secondary task (visual, auditory, visual + auditory; adaptive, non-adaptive). Performance was assessed through mistakes in the tracking task (lateral deviation from the central line). Additionally, the participants were asked for their subjective opinion of this system-driven change of modality presentation. Findings do not support the assumption that changing from bimodal (visual + auditory) to auditory-only presentation under high situational demands leads to best performance in the driving task. Rather, auditory-only information presentation was best under all conditions and led to the fewest mistakes in the tracking task. Moreover, subjective ratings revealed that $50 \%$ of the subjects favoured auditory-only presentation and $75 \%$ of the subjects generally preferred non-adaptive information presentation.
\end{abstract}

\section{DRIVING AS MULTITASKING}

Many vehicle drivers no longer use the car solely to get from one place to another. They may also engage in other activities while driving, for example using navigation systems or answering phone calls. A lot of technology in new vehicles offers assistance to the driver, but on the other hand, it can also be a source of distraction. So driving becomes a matter of dividing attention between the primary task of driving the vehicle and several other subtasks. This kind of multitasking can lead to dangerous situations because drivers continue with in-vehicle tasks even when the situation on the road demands their full attention (Green, 2004). As a consequence, secondary task involvement resulting in driver distraction is a cause of many accidents (see Ranney, 2000). Thus Driver Assistance Systems (DASs) are being developed to avoid overloading the driver's information processing channels and to keep workloads moderate. There are many possible ways to support drivers, and distinctions must be made. Driver assistance systems are concerned with aiding drivers in the completion of the primary driving task, e.g., adaptive cruise control. In contrast, a DAS integrated in information and communication systems does not assist in the primary driving task (Faerber, 2005; Kopf, 2005). The following study deals with design of a situation-dependent change of the presentation 
modality of a secondary task, which was not related to the driving task. According to Wandke (2005) this kind of assistance is termed "presentation assistance."

\section{MULTIPLE RESOURCES AND TIME-SHARING}

The basic assumption in driving a car is that the more demanding the situation, the more load is put on the driver, which should result in a higher workload. Evidence for this thesis exists in cases where driving at high speed led to increased workload in order to drive safely (Godley, 1997). According to Wickens (2002) workload can be defined in terms of time needed to carry out a task in relation to the available time. This explains why coordination and completion of two tasks can be seen as a matter of time-sharing. Moreover, his multiple resource theory (2002) states that having to deal with the driving situation and a secondary task that demands the same resource properties as the primary driving task will lead to a degradation in performance. Resource properties consist of: processing stages, perceptual modalities, visual channels and processing codes (see Figure 1).

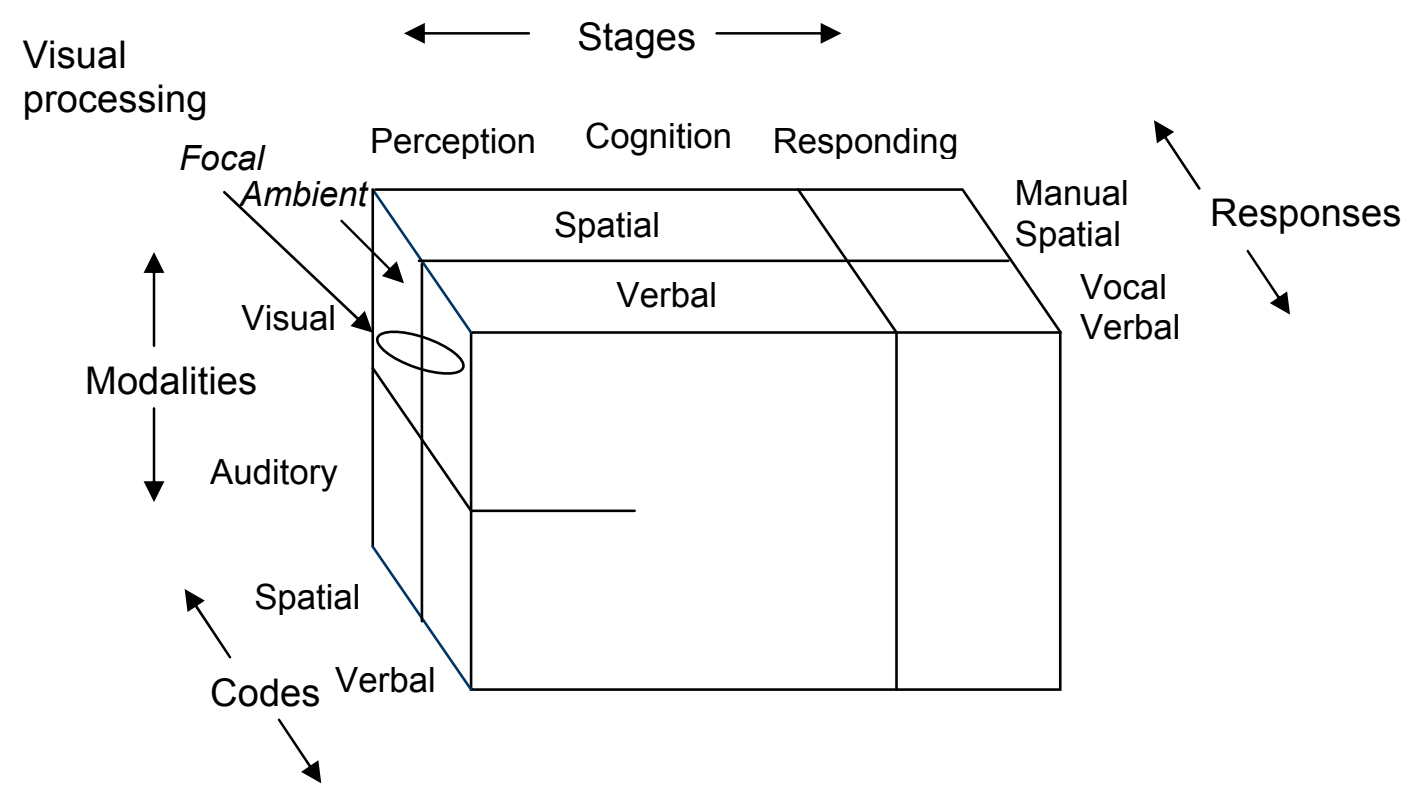

Figure 1. Wickens' multiple-resource theory (2002)

Therefore time-sharing between tasks that involve different modalities is more beneficial than intra-modal time-sharing. For this reason, a DAS that focuses on assistance in secondary tasks, especially in high-demand situations, should be designed carefully concerning the resource properties addressed, so that time-sharing between two tasks becomes easier.

\section{Choosing the best modality}

To determine which modality suits best in a certain situation, one can consider global research findings. In general, visual information presentation is best where the presented information needs to be persistent because one cannot immediately respond to it. Another reason to use visual information communication is when information is long or complex (Bailey, 1996; Belz, 1997). Auditory information presentation, in contrast, is useful if information is short and the person to 
be informed already has to deal with other visually demanding tasks (Bailey, 1996). Using several modalities at once as redundant information presentation can ease processing of information and lead to reduced workload (Hedicke, 2000; Salmen, 2002). Therefore, in a driving situation with simultaneous involvement in a secondary task, we assumed that multimodal information presentation of the secondary task would be useful for successfully completing both tasks except in critical situations when bimodal information presentation is assumed to be too distracting. One idea to handle this problem is to facilitate time-sharing through a DAS that changes its mode of information presentation of the secondary task according to the demands of the situation. The research question is whether performance in the primary task increases, deteriorates or stays the same through this kind of adaptive system behavior compared to non-adaptive modality presentation. Additionally, it is interesting to know how a system-initiated adaptation of modality presentation is perceived and accepted by users.

\section{EXPERIMENTAL SETTING}

Forty-two subjects took part in the study, 22 men and 20 women, with ages between 20-46 years (mean: 29.5), 62\% of them students. They were recruited through a university advertisement and received a reward of 15 Euro. All of them had a driving license. Their main task was to engage in a computer-simulated tracking task, which is a continous task like driving a car, with a steering wheel as normally used in computer games. From time to time they also had to perform a decision-making task, which was to identify special numerical series. The task was an analogy to receiving a phone call while driving.

The independent variables consisted of 1) demands of the situation (low vs. high) and 2) presentation modality of the secondary task (visual, auditory, visual+auditory; adaptive, nonadaptive. The presentation modality of the subsidiary task differed according to the demands of the situation. A less-demanding situation was represented via tracking at low speed compared to a high-demanding situation at increased speed. All participants performed the tracking task under low and high situational demand. With adaptive modality presentation, the presentation modality of the subsidiary task changed simultaneously with a shift in demand of the situation (see Table 1). So, for example, one condition was secondary task presentation visually under low tracking speed and a change to auditory presentation under high-tracking velocity.

Table 1. Adaptive and non-adaptive conditions in the experiment

\begin{tabular}{|c|c|}
\hline Adaptive information presentation & Non-adaptive information presentation \\
\hline change from visual to auditory & consistent visual \\
\hline Change from visual to bimodal (visual + auditory) & consistent auditory \\
\hline change from auditory to bimodal & consistent bimodal \\
\hline change from auditory to visual & \\
\hline change from bimodal to visual & \\
\hline change from bimodal to auditory & \\
\hline
\end{tabular}

Visual information was presented on a second monitor besides the main monitor. A male voice was used for auditory data presentation, and in visual + auditory conditions the series were presented on the second monitor and verbally. Every subject experienced one non-adaptive and two adaptive conditions, one after the other with a break after each. 


\title{
RESULTS
}

Severe and minor mistakes made in the tracking task were recorded, glances to the secondary monitor were gathered for the visual/auditory condition, and questionnaires were used to collect data about the preferences of the subjects regarding the modes and concerning adaptive information presentation. Statistical calculations included data for 42 subjects.

Table 2. MANOVA main-effects for mistakes in the tracking task

\begin{tabular}{|l|c|c|c|}
\hline Effect & $\begin{array}{l}\text { Presentation } \\
\text { Modality }\end{array}$ & Situation & $\begin{array}{l}\text { Presentation Modality } \\
\text { * Situation }\end{array}$ \\
\hline Score & .44 & .12 & .32 \\
\hline F & 1.54 & 97.57 & 2.2 \\
\hline Hypo. Df & 64 & 8 & 64 \\
\hline Rem. Df & 640.96 & 110 & 640.96 \\
\hline Sign. & .01 & .00 & .00 \\
\hline
\end{tabular}

Results in the objective measures showed a significant main effect of the factor "demanding situation" (see Table 2). This meant that driving at high speed resulted in more mistakes in the primary task than driving at low speed. There was also an effect of "presentation modality" and an interaction effect (see Table 2). Further analysis revealed that non-adaptive auditory presentation of the secondary task led to fewest mistakes in the tracking task compared to all other conditions. In addition to these findings, subjective data also demonstrated that about $50 \%$ of all subjects liked the non-adaptive auditory information presentation the most; in general, about $40 \%$ of all subjects felt a change in the presentation mode in general was irritating.

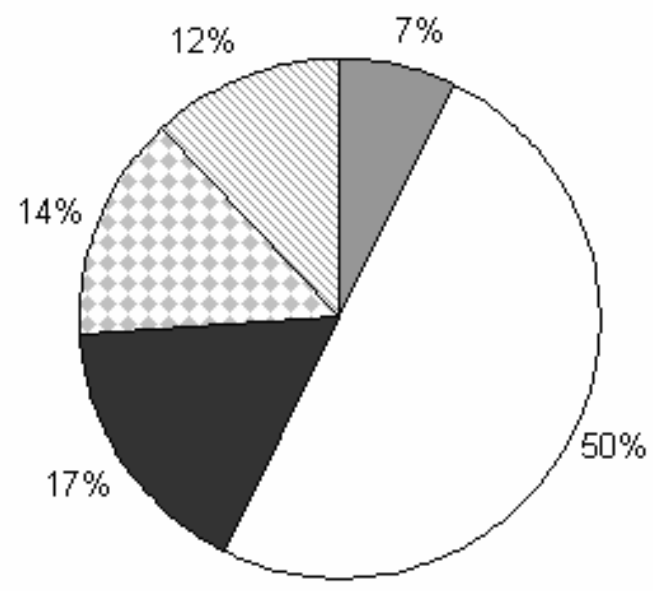

\author{
$\square$ consistent visual \\ $\square$ consistent auditive \\ consistent bimodal \\ change from bimodal to auditive \\ change from visual to auditive
}

Figure 2. Subjective data of preferred modality presentation

Moreover, focusing on the non-adaptive conditions, about $75 \%$ preferred non-changing modality presentation (see Figure 2). These findings do not support the assumption that changing from 
bimodal (visual + auditory) to auditory-only presentation under high situational demands leads to the best performance in the primary driving task.

\section{DISCUSSION}

Besides the results that show an advantage for non-adaptive auditory-verbal information presentation, there were also unexpected negative side effects. Subjects stated that they were irritated and confused about the changes in modality. In addition, most did not realize the relationship between change of speed and simultaneous variation of presentation modality. This lack of transparency of the system actions meant that subjects' expectations did not correspond to system behavior.

Nitschke (2004) reported that, on the basis of an investigation in the context of consumer electronics, adaptive assistance functions were rated worse than non-adaptive assistance functions. Reasons mentioned for this effect were lack of control and absence of trust in system behaviour. Perhaps the non-existence of transparency in our study also evoked feelings of lack of control and this influenced performance, as well as subjective ratings. Lacking trust could have been a contributing factor to the negative side effects, since some subjects mentioned using the visual component in the visual + auditory condition to check if the material presented auditorily and visually was really identical. Subjects in this investigation probably viewed the changing modalities as inconsistently and randomly switching. Research claimed that inconsistency and lack of experience can lead to higher workload (Verwey, 1993), and this in turn could have been one reason why no positive effects of adaptive modality changing could be shown and the reported negative side effects occurred. The system behaviour, therefore, could not lead to building a correct mental model of the functioning of the DAS.

Another finding was observed in terms of recorded number of glances to the secondary monitor where the secondary task was presented. It was found that the visual component in the visual + auditory condition was used less when tracking at high speed. This means that under high workload conditions subjects adapted their behavior to the changed circumstances of the situation. This effect is in contrast to the findings of a similar study (Sauer \& Polkehn, 2003). In their study, the presentation of a navigation system varied according to the demands of the situation. Navigation assistance was presented through an arrow as a visual component plus speech in low-demand situations, and by speech only in high-demand situations. It was found that subjects tended to use the visual component more in high-demand situations, which were defined as high speed and poor visibility. This is probably one reason for the differences found in viewing behaviour compared to this study, where there was no need to compensate for low visibility by glancing to the secondary monitor. The specific task involved is very relevant, since navigation information might benefit from visual presentation, whereas infotainment information as investigated in this study does not benefit from a visual presentation. That might be why people behaved reasonably and did not use the visual component in low-demand situations.

Impeding a secondary task such as receiving a phone call at higher driving speeds is more reasonable because the visual-distraction does not seem to be the leading factor in such highworkload situations. Piechulla et al. (2003) used a similar approach through a workload estimator, which directed incoming phone calls to the mailbox in high-workload situations. As a 
tendency, they reduced the drivers' mental workloads, and it should be further explored whether this also promotes performance in the primary driving task.

Finally one can state that an adaptive change from bimodal to auditory-only presentation of the secondary task, which was expected provide the best assistance in avoiding overloading the information processing channels at higher workloads, did not support an improvement in primary task performance. Moreover, there were acceptance problems for verbal statements in terms of confusion about this kind of adaptive system behaviour. The results of this study provide no support for adaptive DAS.

\section{ACKNOWLEDGMENTS}

This study was part of a research cooperation project between the Robert Bosch $\mathrm{GmbH}$ and DUBROWSKY \& PARTNER. Others who contributed to this work are Andreas Dubrowsky, Joachim Warning, Dr. Guido Beier and Sven Wittenberg.

\section{REFERENCES}

Bailey, R.W. (1996). Human performance engineering. New Jersey: Prentice Hall.

Belz, S.M. (1997). A simulator-based investigation of visual, auditory, and mixed-modality display of vehicle dynamic state information to commercial motor vehicle operators. Unpublished Master's Thesis. Virginia Polytechnic Institute and State University, Blacksburg, VA. Available online: http://scholar.lib.vt.edu/theses/available/etd-11297195327/unrestricted/thesis.pdf [11/12/06]

Färber, B. (2005). Erhöhter Fahrernutzen durch Integration von Fahrerassistenz- und Fahrerinformationssystemen, in Maurer M. \& Stiller C. (Eds.), Fahrerassistenzsysteme mit maschineller Wahrnehmung. Berlin: Springer, 141-160.

Fussel, S.R., Grenville, D., Kiesler, S., Forlizzi, J., \& Wichansky, A. (2002). Accessing multimodal information on cell phones while sitting and driving. Proceedings of the Human Factors and Ergonomics Society. Available online: http://www-2.cs.cmu.edu/ sfussell/ pubs/Manuscripts/HFES02-CellPhones-Final.pdf [11/12/06]

Godley, S.T. (1999). A driving simulator investigation of perceptual countermeasures to speeding. PhD Thesis: Monash University. Available online: http://www.psych.usyd.edu.au/staff/stuartg/GodleyThesis.pdf [11/12/06]

Green, P. (2004). Driver Distraction, Telematics Design, and Workload Managers: Safety Issues and Solutions. SAE Paper Number 2004-21-0022. Available online: http://www.umich.edu/ driving/publications/GreenConvergence04paper4b.pdf [11/12/06]

Hedicke, V. (2000). Multimodalität in Mensch-Maschine-Schnittstellen. In K.-P. Timpe, T. Jürgensohn \& H. Kolrep (Eds.), Mensch-Maschine-Systemtechnik. Düsseldorf: Symposion, 203-229.

Kopf, M. (2005). Was nützt es dem Fahrer, wenn Fahrerinformations- und -assistenzsysteme etwas über ihn wissen? In Maurer, M., Stiller C., (Eds.), Fahrerassistenzsysteme mit maschineller Wahrnehmung. Berlin: Springer, 117-140. 
Nitschke, J. (2004). Assistenz bei Auswahlprozessen - Untersuchungen zu adaptiver und adaptierbarer Assistenz bei der Auswahl von Filmen. PhD Thesis: Berlin.

Piechulla, W., Mayser, C., Gehrke, H., \& König, W. (2003). Reducing drivers' mental workload by means of an adaptive man-machine interface. Transportation Research Part F, 6, 233248.

Ranney, T.A., Mazzae, E., Garrot, R. \& Goodman, M.J. (2000). NHTSA driver distraction research: Past, present, and future. Available online: http://www-nrd.nhtsa.dot.gov/ departments/nrd-13/driver-distraction/PDF/233.PDF [11/12/06]

Salmen, A. (2002). Multimodale Menüausgabe im Fahrzeug. PhD Thesis: Regensburg.

Sauer, J. \& Polkehn, K. (2003). Durchführung von Nutzerevaluationen eines Navigationssystems aus dem Kraftfahrzeugbereich. Bericht zur Evaluation des KFZ-Demonstrators in Phase II: adaptive Navigationsassistenz.

Verwey, W.B. (1993). How can we prevent overload of the driver? In A. M. Parkes \& S. Franzén (Eds.), Driving future vehicles. London: Taylor \& Francis, 235-244.

Wandke, H. (2005). Assistance in human-machine interaction: a conceptual framework and a proposal for a taxonomy. Theoretical Issues in Ergonomics Scienc, 6, 129-155.

Wickens, C.D. (2002). Multiple resources and performance prediction. Theoretical Issues in Ergonomics Science, 3 (2), 159-177. 